\title{
Aspectos da biologia de Buenoa platycnemis (Fieber) (Insecta, Heteroptera, Notonectidae) da Restinga de Maricá, Rio de Janeiro
}

\author{
Jorge Luiz Nessimian \\ José Ricardo Inacio Ribeiro ${ }^{1}$
}

\begin{abstract}
On the biology of Buenoa platycnemis (Fieber) (Insecta, Heteroptera, Notonectidae) at Restinga de Maricá, Rio de Janeiro State, Brazil. A monthly quantitative study on B. platycnemis (Fieber, 1851) was carried out in a sand dune marsh, on the littoral of Rio Janeiro State. The aims of this study were to correlate the life cycle of the species with the seasonal regime of the water body, defining steps of the annual cycle, age structure, and to indicate oviposition sites and trophic relations. The species shows a marked seasonal tendency. The major steps of the life cycle obtained were an invernal (weak) with a predominance of imagines, followed by another of population expansion, characterized by the absence of adults, massive eclosions and predominance of low instar nymphs. Probably, B. platycnemis has a sensibility to water level variation and might be influenced by the concentration of Spirogyra sp. (Chlorophyceae) in the water column. In relation to oviposition, $B$. platycnemis has not shown preference for any macrophyte, since eggs were found in Rhynchosphora corymbosa (Linnaeus) Britton (Cyperaceae), Eleocharis sellowiana Kunth. (Cyperaceae) and Nymphoides indica (Linnaeus) O. Kuntze. (Menyanthaceae). The observed predators of B. platycnemis were Anax amazilli (Burmeister, 1839) (Aeshinidae), Erythemis credula (Hagen, 1861) (Libellulidae) and other Odonata, nymphs and adults of Belostoma candidulum Montandon, 1903 (Belostomatidae), Notonecta (Paranecta) disturbata Hungerford, 1926, Buenoa antigone antigone (Kirkaldy, 1899) (Notonectidae), besides Curicta cf. tibialis (Martin, 1898) and Ranatra horvathi Montandon, 1910 (Nepidae). Larvae of Sayomyia sp. (Chaoboridae), Chironomus spp. and Labrundinia sp. (Chironomidae), Callibaetis guttatus Navás, 1915 (Baetidae), cladocerans and copepods are potential prey of B. platycnemis, while ostracodes were refused.
\end{abstract}

KEY WORDS. Nepomorpha, Notonectidae, Buenoa platycnemis, marsh, restinga

As espécies de Buenoa Kirkaldy, 1904 (Notonectidae) podem ser encontradas em poças e lagos de água doce, sendo que muitas podem ou não habitar corpos d'água com vegetação aquática (TRUXAL 1953). Segundo GitTELMAN (1975), Buenoa spp. são encontradas em uma variedade de poças de água doce com temperaturas relativamente altas. A maioria das espécies é predadora efetiva de larvas de dípteros, microcrustáceos e nematódeos. BARE (apud TRUXAL 1953) observou cloroplastos de Spirogyra sp. (Chlorophyceae) no intestino médio de algumas espécies, constatando também

1) Departamento de Zoologia, Instituto de Biologia, Universidade Federal do Rio de Janeiro. Caixa Postal 68044, 21944-970 Rio de Janeiro, Rio de Janeiro, Brasil.

E-mail: nessimia@acd.ufrj.br

Revta bras. Zool. 17 (1): 229 - 239, 2000 
a utilização de algas quando outra fonte de alimento não está disponível. Algumas espécies preferem presas que apresentam maior mobilidade através da coluna d'água, tornando-se facilmente disponíveis (GITTELMAN 1975).

Em estudos realizados em diversos corpos d'água na Restinga de Maricá por RIBEIRO et al. (1998), acerca dos Nepomorpha, Buenoa platycnemis (Fieber, 1851) (Notonectidae) foi a espécie dominante. Os resultados desses estudos mostraram existir variação populacional devido à influência do tamanho e do nível de água do criadouro colonizado. Os Notonectidae foram os mais influenciados, apresentando relação direta com o aumento de seston e, conseqüentemente, plâncton (RIBEIRO et al. 1998).

A oviposição, nas espécies de Buenoa, é endofítica, sendo utilizadas diversas macrófitas aquáticas. O padrão estrutural do cório, o formato e o tamanho dos ovos foram bem estudados, possibilitando a identificação de algumas espécies por essas estruturas (RICE 1954).

O presente trabalho objetiva relacionar o ciclo de vida de B. platycnemis ao regime sazonal do Brejo-canal de Itaipuaçú, definindo etapas acerca do ciclo anual e a estrutura etária desta espécie, indicando os sítios de oviposição e suas relações tróficas nesse ambiente temporário.

\section{Área de estudo}

O estudo se desenvolveu na Restinga de Maricá, no município de Maricá, estado do Rio de Janeiro ( $\left.22^{\circ} 57^{\prime} \mathrm{S}, 43^{\circ} 54^{\prime} \mathrm{W}\right)$. A região enquadra-se no clima Aw de Köppen, com verão chuvoso e inverno seco. A temperatura média anual varia entre 22 e $24^{\circ} \mathrm{C}$ e a precipitação média anual, entre $1.000 \mathrm{~mm}$ e $1.350 \mathrm{~mm}$ (FRANCO et al. 1984).

O Brejo-canal de Itaipuaçú, com área aproximada de $150.000 \mathrm{~m}^{2}$, situa-se na depressão que separa os dois cordões arenosos (dunas), sendo sua formação resultado do fechamento do canal de mesmo nome há 43 anos (Oliveira et al. 1955). Suas águas são escuras e ácidas e o substrato de fundo consiste de uma espessa camada de matéria orgânica (turfa), recobrindo o solo arenoso. O nível de água no brejo é dependente da flutuação do lençol freático e apresenta quatro fases distintas: seca, enchente, cheia e vazante (CARMO \& LACERDA 1984). O ciclo hidrológico é acompanhado por mudanças nas condições químicas, físicas e físico-químicas da água. Fauna e flora apresentam adaptações (fisiológicas, morfológicas e comportamentais) ao caráter temporário do corpo d'água, tendo diversas espécies, ciclo de vida congruente ao ciclo hidrológico (NESSIMIAN 1995a). Vizinhas ao brejo, ocorrem diversas poças, também temporárias, cujo nível d'água depende também das chuvas e que apresentam leito arenoso ou argiloso (RIBEIRO et al. 1998).

O brejo apresenta sua superfície grandemente ocupada por macrófitas, sendo as principais: Eleocharis sellowiana Kunth. (Cyperaceae), Utricularia gibba Linnaeus (Lentibulariaceae), Rhynchosphora corymbosa (Linnaeus) Britton (Cyperaceae), Nymphoides indica (Linnaeus) O. Kuntze. (Menyanthaceae) e Sagitaria lancifolia sbps lancifolia Bog. (Alismataceae). Proliferações de Spirogyra sp. ocorrem nos períodos de enchente e cheia. 


\section{MATERIAL E MÉTODOS}

O material estudado é proveniente de amostragens quantitativas mensais, realizadas em dois períodos, de janeiro de 1987 a fevereiro de 1988 e de janeiro a junho de 1996. No primeiro período, as coletas foram realizadas com amostradores de bentos de diâmetro de $30 \mathrm{~cm}$ e malha de $400 \mu \mathrm{m}$ de abertura (NESSIMIAN 1995a), sendo o número de amostras, 15 para cada mês. As coletas do segundo período foram efetuadas com peneiras de aproximadamente $0,246 \mathrm{~m}^{2}$ e malha de $1 \mathrm{~mm}$ de abertura, sendo 80 amostras por mês.

As espécies de macrófitas encontradas foram identificadas por Ana Angélica Monteiro de Barros (Instituto de Química, UFF). Estas foram observadas quanto à sua distribuição no brejo e à presença de posturas de B. platycnemis. Valores estimados de densidade de Spirogyra sp., referentes ao primeiro período amostral, foram extraídos de DA SILVA (1998). Mensalmente, foram tomados valores de temperatura da água e do ar (medidos no momento da coleta), do oxigênio dissolvido através do método de Winkler (BROWER \& ZAR 1977) e do pH, em ambos os períodos amostrais. Valores de condutividade elétrica e de concentração de nutrientes (nitrito, fosfato e amônia) foram tomados somente para o segundo período amostral. As titulações foram realizadas no Laboratório de Avaliação Ambiental, Departamento de Biologia Marinha, Universidade Federal do Rio de Janeiro. Dados médios mensais de pluviosidade para Maricá foram fornecidos pela Superintendência Estadual de Rios e Lagoas e pela Agência Nacional de Energia Elétrica.

Associações entre o número de indivíduos da espécie em todos os meses de coleta e entre os valores obtidos para cada uma das variáveis ambientais foram inferidas através da regressão múltipla (forward stepwise) (SOKAL \& ROHLF 1969). Para comparações entre os meses, quanto à distribuição espacial da espécie utilizou-se o teste do $X^{2}$ e quanto à densidade populacional, a correlação de Pearson (ElliotT 1977). Dados de relações tróficas foram obtidos através de observação (em campo e laboratório) e análise do conteúdo digestivo de espécies potencialmente predadoras.

A estrutura etária da população de B. platycnemis foi verificada mensalmente através da separação dos estádios de desenvolvimento, definindo, analogamente ao estudo de SCHNACK et al. (1979) com Belostomatidae, etapas em seu regime populacional.

\section{RESULTADOS}

\section{Fatores abióticos}

A pluviosidade média mensal foi de $131,4 \mathrm{~mm}$ em 1987, com valores máximo e mínimo, respectivos, em maio e julho. Nos dois meses amostrados em 1988 , o total de chuvas somou $601,4 \mathrm{~mm}$, sendo $511,4 \mathrm{~mm}$ somente em fevereiro. Em 1996, a média dos primeiros seis meses foi de 113,9 mm, não havendo variação significativa. No primeiro período amostral, as profundidades máxima e mínima do brejo ocorreram, respectivamente, em junho e janeiro de 1987. Contudo, em 1996, o mínimo verificado em março corresponde provavelmente a uma recessão eventual no período de subida do nível d'água. $\mathrm{O} \mathrm{pH}$ variou pouco, ao redor de 5,0 . Valores máximos ocorreram durante a descida do nível de água e na vazante enquanto que 
os menores, nos períodos de subida e de cheia. Embora com medidas pontuais, a temperatura da água mostrou o padrão esperado com valores maiores nos meses de verão e menores nos de inverno. A variação na concentração de oxigênio dissolvido foi similar à do nível de água em 1987. No período de 1996, embora o menor valor tenha ocorrido no mês de janeiro, a concentração de oxigênio manteve-se estável em torno de $68 \%$. Os valores de condutividade elétrica medidos no segundo período amostral (1996) mostraram-se dentro do padrão esperado para o corpo d'água, com um pico no início da subida do nível d'água e uma diminuição gradativa em direção à cheia, refletindo, porém, a pequena variação do nível d'água nesse período (Tab. I).

Tabela I. Medidas de precipitação para Maricá e variáveis ambientais medidas mensalmente no Brejo-canal de Itaipuaçú em dois periodos amostrais (janeiro 1987 a fevereiro 1988 e janeiro 1996 a junho 1996). (ND) Não determinado.

\begin{tabular}{|c|c|c|c|c|c|c|c|c|c|c|c|c|}
\hline \multirow{2}{*}{ Parâmetros } & \multicolumn{12}{|c|}{1987} \\
\hline & Jan & Fev & Mar & $\mathrm{Abr}$ & Mai & Jun & Jul & Ago & Set & Out & Nov & Dez \\
\hline Profundidade $(\mathrm{cm})$ & 12,1 & 26,8 & 29,2 & 46,0 & 45,3 & 54,2 & 54,2 & 46,5 & 39,7 & 37,5 & 34,2 & 30,1 \\
\hline Temperatura $\left({ }^{\circ} \mathrm{C}\right)$ & 32,3 & 38,0 & 26,2 & 30,0 & 29,0 & 28,0 & & 25,5 & & 33,0 & 28,0 & 28,0 \\
\hline & 5,0 & 5,0 & 5,0 & 4,5 & 4,5 & 4,5 & & 4,7 & 5,1 & 4,9 & 5,7 & 5,5 \\
\hline Oxigênio dissolvido (\%) & & 44,2 & 61,7 & 69,8 & 99,7 & 95,5 & & 122,0 & 99,2 & 87,0 & 22,9 & 16,0 \\
\hline \multirow[t]{3}{*}{ Precipitaçăo (mm) } & 87,3 & 187,7 & 102,4 & 207,5 & 227,2 & 272,0 & 23,6 & 57,2 & 129,4 & 116,6 & 54,4 & 224,4 \\
\hline & \multicolumn{2}{|c|}{1988} & \multicolumn{6}{|c|}{1996} & & & & \\
\hline & Jan & $\overline{\mathrm{Fev}}$ & Jan & Fev & Mar & $\mathrm{Abr}$ & Mai & $\overline{\text { Jun }}$ & & & & \\
\hline Profundidade $(\mathrm{cm})$ & 14,9 & 37,0 & 35,6 & 30,7 & 24,30 & 28,50 & 40,3 & 45,30 & & & & \\
\hline Temperatura $\left({ }^{\circ} \mathrm{C}\right)$ & 33,0 & 28,0 & 33,3 & 33,3 & 35,60 & 27,70 & 27,0 & 24,30 & & & & \\
\hline $\mathrm{pH}$ & 5,2 & 5,5 & 5,6 & 5,0 & 4,60 & 4,80 & 4,30 & 5,00 & & & & \\
\hline Oxigẽnio dissolvido (\%) & 11,0 & 29,0 & 26,5 & 67,0 & 79,00 & 56,30 & 68,80 & 69,80 & & & & \\
\hline Condutividade elétrica $\left(\mu \mathrm{S} . \mathrm{cm}^{-1}\right)$ & - & - & 258,3 & 154,71 & 160,70 & 162.0 & 150,70 & 121,30 & & & & \\
\hline Amonia $\left(\mu \mathrm{mol} . \mathrm{cm}^{-1}\right)$ & - & - & 4,76 & ND & 3,58 & 0,05 & 24,87 & 0,48 & & & & \\
\hline Nitrito $\left(\mu \mathrm{mol} . \mathrm{cm}^{-1}\right)$ & - & - & 38,23 & ND & 0,28 & 0,19 & 0,25 & ND & & & & \\
\hline Fosfato $\left(\mu \mathrm{mol} . \mathrm{cm}^{-1}\right)$ & - & - & 0,20 & ND & 0,34 & 0,03 & 0,13 & 0,03 & & & & \\
\hline Precipitaçăo (mm) & 90,0 & 511,4 & 113,90 & 125,11 & 119,70 & 108,50 & 85,40 & 130,50 & & & & \\
\hline
\end{tabular}

\section{Ciclo populacional de B. platycnemis, sítios de postura e relações com} o meio

Buenoa platycnemis ocorreu durante praticamente todo o período amostral, exceto em fevereiro de 1987 e de 1988, com maior concentração nos meses de primavera em 1987 (176,37 ind. $\mathrm{m}^{-2}$ em novembro). Neste ano, os adultos figuraram nas amostras a partir de abril, porém ninfas de último estádio estiveram presentes desde janeiro. Ninfas menores ocorreram a partir de abril e, como toda a população, apresentaram incremento em sua freqüência nos meses de setembro a novembro, quando a espécie foi observada em abundância no brejo (Fig. 1). No período de aumento da população, foram obtidos valores muito altos do $X^{2}(141 ; \mathrm{gl}=14 ; \mathrm{p}<$ 0,001 ; distribuição contagiosa). Provavelmente, a predominância de indivíduos mais jovens foi o fator responsável por esse aumento. Além disso, a baixa concentração dessa população em janeiro de 1988 também explica o alto valor do $X^{2}$ (218; $\mathrm{gl}=14 ; \mathrm{p}<0,001)$. As coletas realizadas em 1996 mostraram também que a espécie apareceu em todas as amostras, obtendo os maiores valores em janeiro $\left(8,59\right.$ ind. $\left.\mathrm{m}^{-2}\right)$ e maio $\left(8,33\right.$ ind. $\left.\mathrm{m}^{-2}\right)$. Neste periodo amostral, os adultos estiveram presentes em todos os meses de coleta. As ninfas de primeiro estádio só ocorreram em fevereiro e maio, enquanto que ninfas de segundo e quarto estádios não apareceram. Ninfas 
de terceiro estádio mantiveram sua freqüência relativamente constante. A menor concentração encontrada foi em março e a maior em junho. As ninfas de quinto estádio obtiveram seu pico populacional em maio, enquanto sua menor concentração foi obtida em fevereiro. Comparando-se as curvas de densidade da espécie de meses correspondentes nos dois períodos de coleta, nota-se uma correlação positiva, embora não significativa $(r=0,48, p=0,167)$.
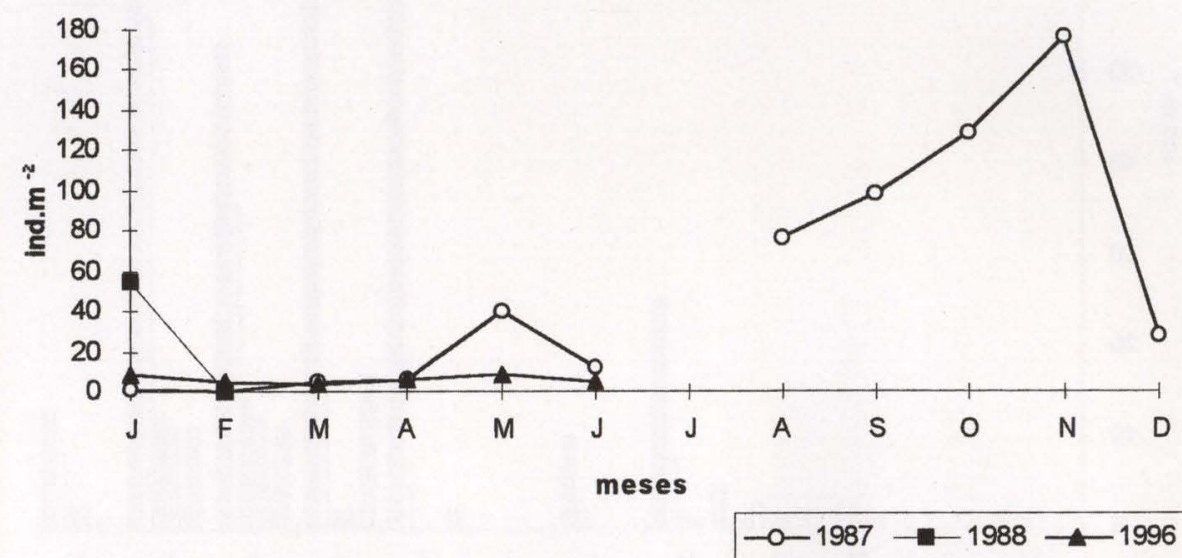

Fig. 1. Variação de freqüência de Buenoa platycnemis durante dois periodos amostrais (janeiro 1987 a fevereiro 1988 e janeiro 1996 a junho 1996) no Brejo-canal de Itaipuaçú, Maricá, Rio de Janeiro.

SCHNACK et al. (1979) caracterizaram quatro períodos distintos no ciclo anual de uma espécie de Belostomatidae: uma etapa de expansão, com eclosões massivas e predomínio de estádios mais jovens; uma etapa estival, caracterizada pela escassa predominância de um estádio particular sobre os restantes; uma etapa de declínio dos estádios inferiores, com uma elevada freqüência dos estádios superiores e de adultos; uma etapa invernal, compreendendo um período longo com o predomínio de adultos e a escassez de ninfas de quarto e quinto estádios. Utilizando o modelo acima para B. platycnemis, os resultados obtidos nos dois períodos mostram que a espécie apresenta uma etapa invernal não muito definida (de junho até setembro), com o predomínio de adultos sobre os outros estádios e o gradativo aumento no número de ninfas de quinto estádio, o que não foi observado no modelo. Essa etapa é seguida de outra, caracterizada pela expansão da população e ausência de adultos, eclosões massivas e predominância de estádios muito jovens. Essa etapa vai de setembro até janeiro, principalmente. Não ficou clara a presença da etapa estival e de declínio dos estádios inferiores, que ocorreu de março a junho. O leve declínio de adultos de janeiro a março de 1996 confirma a emigração massiva no período de verão, coincidindo com a etapa de expansão caracterizada acima. Mas, provavelmente, devido a limitações inerentes ao método de coleta usado neste período, não foi possível detectar com clareza a presença das ninfas muito jovens oriundas das eclosões que provavelmente estão presentes, como ocorreu em janeiro de 1988 (Fig. 2). 


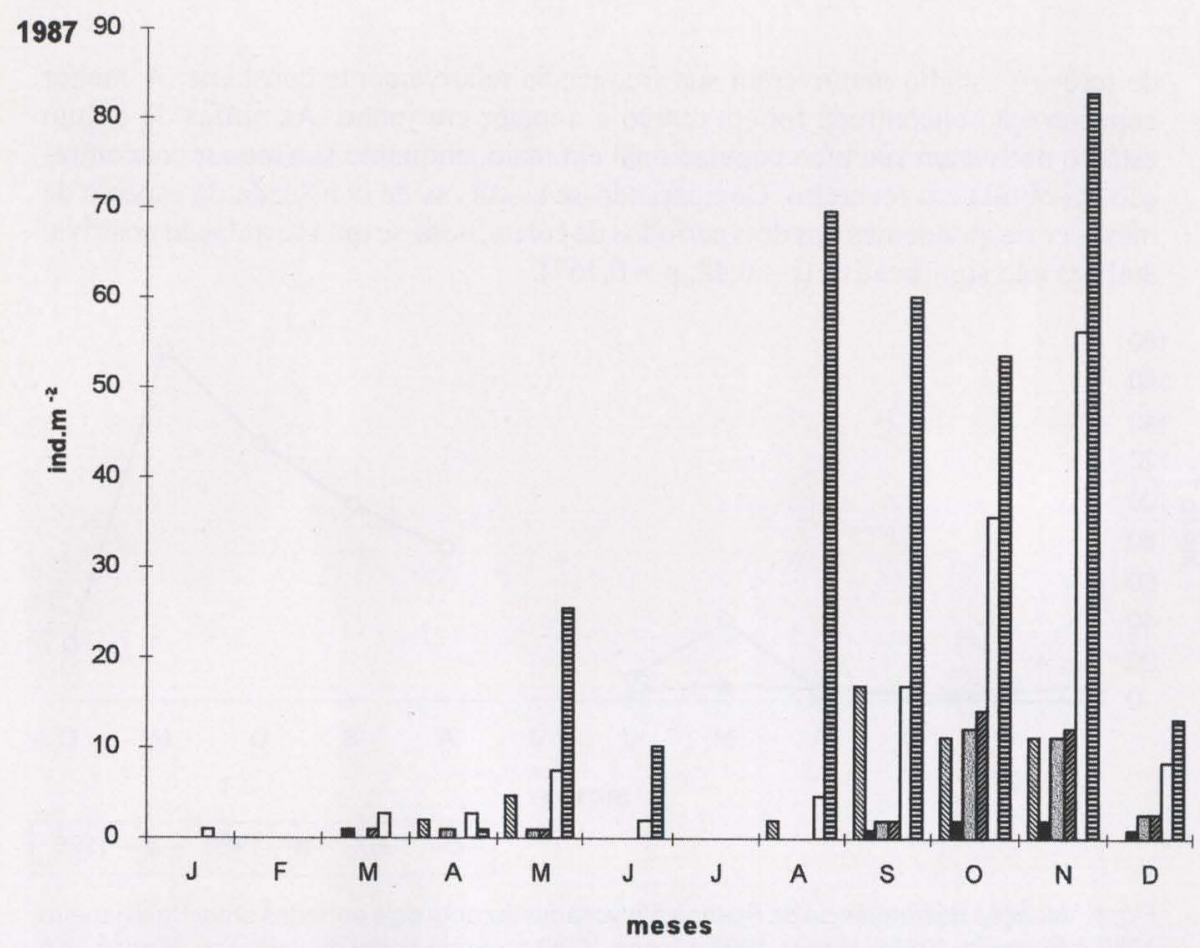

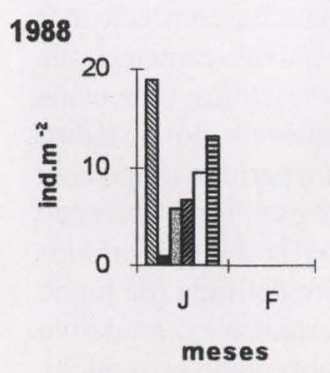

⿶1
- N2
1996

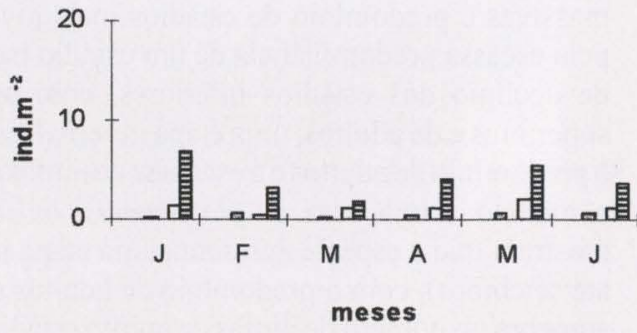

N44
口N5

Fig. 2. Estrutura etária de Buenoa platycnemis durante dois periodos amostrais (janeiro 1987 a fevereiro 1988 e janeiro 1996 a junho 1996) no Brejo-canal de Itaipuaçú, Maricá, Rio de Janeiro. (N1, N2, N3, N4, N5) Ninfas de primeiro a quinto estádios, respectivamente.

Os resultados da regressão múltipla, tendo como variáveis independentes a quantidade de Spirogyra sp., o pH, a profundidade, oxigênio dissolvido e temperatura, mostram que somente houve correlação significativa da espécie com Spirogyra sp. (Tab. II). 
Tabela II. Principais valores da regressão linear múltipla do número total de indivíduos de Buenoa platycnemis no Brejo-canal de Itaipuaçú, Restinga de Maricá, Rio de Janeiro, em relação à quantidade de algas do gênero Spirogyra e pH.

\begin{tabular}{lcccc}
\hline & Correlaçăo parcial & $p$ & Múltiplo $\mathrm{R}^{2}$ & Variação $\mathrm{R}^{2}$ \\
\hline Spirogyra & 0,9656 & 0,0003 & 0,9323 & - \\
$\mathrm{pH}$ & 0,1168 & 0,3243 & 0,9454 & 0,0131 \\
\hline
\end{tabular}

Foram encontradas posturas de B. platycnemis em Rhynchosphora corymbosa, Eleocharis sellowiana e Nymphoides indica, plantas abundantes no brejo. Os ovos das espécies de Buenoa são muito similares em tamanho e formato. Próximo à região cefálica, há uma estrutura em forma de boné que se perde freqüentemente quando o ovo é retirado de macrófitas (RICE 1954).

Quanto à alimentação, as observações realizadas em campo e em laboratório mostraram que larvas de Sayomyia Coquillet, 1903 (Chaoboridae), Chironomus Meigen, 1803 e Labrundinia Fittkau, 1962 (Chironomidae) e Callibaetis guttatus Navás, 1915 (Baetidae) são potenciais presas para B. platycnemis. Cladóceros e copépodos também foram predados, enquanto que ostrácodos foram rejeitados. Os predadores de B. platycnemis observados foram Anax amazilli (Burmeister, 1839) (Aeshinidae), Erythemis credula (Hagen, 1861) (Libellulidae) e outras espécies de Odonata. Ninfas e adultos de Belostoma candidulum Montandon, 1903 (Belostomatidae), Notonecta (Paranecta) disturbata Hungerford, 1926, Buenoa antigone antigone (Kirkaldy, 1899) (Notonectidae), além de Curicta cf. tibialis (Martin, 1898) e Ranatra horvathi Montandon, 1910 (Nepidae) também figuraram como predadores efetivos dessa espécie.

\section{DISCUSSÃO}

Em contraste com o modelo proposto por SCHNACK et al. (1979), as etapas do ciclo populacional de $B$. platycnemis, com exceção da etapa de expansão, não foram observadas nitidamente ou com a mesma intensidade e duração. A etapa invernal apresentou-se restrita a um período muito curto, enquanto que as etapas estival e de declínio dos estádios inferiores não ficaram claras nos resultados obtidos. A ausência de amostragem em julho de 1987 dificulta uma conclusão mais precisa. Provavelmente, a menor amplitude de mudanças estacionais em Maricá, quando comparada à da região onde SCHNACK et al. (1979) realizaram seu estudo deve propiciar uma sobreposição das etapas populacionais, como ocorreu entre fevereiro e junho. Mais importante que o gradiente inverno - verão é a natureza temporária do corpo d'água e, variações entre as etapas do ciclo de vida devem corresponder a variações no ciclo hidrológico (NESSIMIAN 1995b; WiGGINS et al. 1980).

A expectativa de que houvesse uma relativa sensibilidade da espécie à variação do nível de água não foi confirmada pelas análises realizadas. Em estudo anterior realizado por RIBEIRO et al. (1998) em diversos corpos d'água de diferentes tamanhos na mesma região, tal relação foi encontrada. Contudo, no Brejo-canal de Itaipuaçú, devido à sua extensão, a flutuação do nível de água, por si só, não acarretou uma resposta imediata da população de B. platycnemis. Provavelmente, as variações populacionais estão relacionadas a outros fatores cíclicos, de alguma 
forma, em função da variação do nível de água. Buenoa platycnemis apresentou correlação positiva significativa com a proliferação de Spirogyra sp., no primeiro período de coleta. Uma grande quantidade de Spirogyra sp. na coluna d'água foi observada no período de maior abundância da espécie (78\% dos indivíduos). Segundo RIBEIRO et al. (1998), a vegetação parece ser um direcionador nas flutuações, ocorrência e distribuição dos indivíduos da espécie. A massa de algas, além de ser usada como outra fonte de alimento, provavelmente também fornece abrigo contra predação (NESSIMIAN 1995a). A espécie ainda apresentou correlação negativa, embora não significativa, com a temperatura da água. A medida que o nível de água do brejo decresce, maiores são as temperaturas alcançadas. Também é nesse período que aumenta a quantidade de litter de macrófitas e algas na coluna d'água, o que acaba impedindo a sua mobilidade e se refletindo no decréscimo populacional que é notado a partir de novembro. Nesse período, há também grande concentração da fauna na coluna d'água propiciando um aumento na predação. Segundo WigGINs et al. (1980), espécies predadoras começam a migrar de ambientes temporários para ambientes permanentes quando termina a primavera e começa o verão (os corpos d'água começam a secar nessa estação). Assim, há provavelmente uma estratégia para evitar a dessecação; além disso, como a oviposição e eclosão são dependentes da água, esses animais colocam ovos no brejo pouco antes de migrar. Dessa forma, eles contribuem para a recolonização desse corpo d'água, que começa a encher no meio do verão, quando os ovos eclodem.

Quanto à postura, a espécie não parece apresentar preferência por uma ou outra macrófita para oviposição (RIBEIRO et al. 1998), o que parece ser vantajoso para uma espécie colonizadora de ambientes temporários.

Segundo CARMO \& LACERDA (1984), são caracterizados quatro períodos limnológicos distintos para o brejo estudado: seca de verão, havendo exposição do solo ao ar atmosférico; nível ascendente de outono; nível alto de inverno; e nível descendente de primavera. A população de B. platycnemis parece possuir uma tendência estacional definida (quando o brejo seguiu esse regime). Os resultados de 1996 mostram que o comportamento populacional da espécie pode mudar de acordo com o regime hídrico do brejo, visto que nos meses de verão, adultos ocorreram em grande proporção e o brejo não secou.

A grande concentração de adultos, ninfas de quinto estádio e um maior número de ninfas de primeiro estádio deve estar relacionada com a tendência desses indivíduos a acasalarem-se na época de cheia do brejo, entre o inverno e a primavera. Isso evidencia um grau de adaptação a esse criadouro, pois o incremento de ninfas de primeiro estádio (oriundo de eclosões contínuas desde janeiro) e de ninfas mais maduras (de gerações anteriores) culmina com o período mais satisfatório para a espécie: o de maior quantidade de nichos disponíveis (NESSIMIAN 1995a). SCHNACK et al. (1979) também obtiveram resultados muito parecidos com os obtidos para $B$. platycnemis ou seja, uma grande quantidade de adultos no inverno.

No primeiro período de coleta, a baixa frequeência de ninfas jovens no verão, principalmente ninfas de primeiro estádio, deve estar relacionada com a seca extrema do brejo em dezembro de 1986, quando houve queima de parte da vegetação. Provavelmente foram inviabilizadas muitas posturas dessa espécie (NES- 
SIMIAN 1995a). Como espécie nectônica, ressalta-se uma conseqüência direta da variação da coluna d'água: retração e expansão de área e profundidade mostram alguma relação com a freqüência da espécie. Em 1996, nos períodos mais quentes, a concentração dessa espécie foi maior quanto menores a profundidade e área do brejo. No período de cheias, o contrário ocorreu (RIBEIRO et al. 1998). De acordo com NESSIMIAN (1995a), a variação da coluna d'água pode influenciar o número de encontros predador - presa. Buenoa platycnemis provavelmente encontra novos tipos de presas e predadores quando o nível da coluna d'água varia. Espécies como $B$. antigone antigone, $N$. (P.) disturbata, Belostoma sanctulum e os Nepidae devem tornar-se predadores mais efetivos ainda, quando o nível da coluna d'água diminui (RIBEIRO et al. 1998) pois B. platycnemis se torna mais disponível devido às características intrínsecas da espécie; ou seja, maior mobilidade e passagem em grande freqüência pela coluna d'água.

SCHNACK et al. (1987) sugere que algumas espécies de Belostoma, devido à sua ausência temporal, são espécies oportunistas, de grande amplitude dispersiva e habitantes típicas de criadouros temporários. Buenoa platycnemis aparentemente possui um comportamento muito semelhante ao descrito acima.

No que concerne à alimentação, GITTELMAN (1975) mostrou que espécies de Buenoa apresentam preferências por espécies com maior mobilidade na coluna d'água. ElLIS \& BORDEN (1970) sugerem que a mobilidade (movimento espacial relacionado à alimentação, respiração etc.) e a motilidade (a habilidade de escapar da captura) são importantes fatores para a seleção das presas por alguns Notonectidae. Segundo ZALOM (1978), as espécies de Buenoa mostram maior tempo de manuseio com a presa do que as de Notonecta. Conseqüentemente, a taxa de predação é mais baixa e o uso da presa é mais eficiente. Holling apud ZALOM(1978) sugere que o tempo de manuseio da presa afeta a predação devido ao decréscimo de tempo disponível para a procura ativa. As observações obtidas em campo e laboratório mostram que organismos com maior mobilidade no necton foram os mais freqüentemente predados. Contudo, não foram feitos testes de preferência alimentar. GitTELMAN (1975) mostra que para algumas espécies de Buenoa, larvas de Diptera e outros Notonectidae estão em primeiro lugar em sua dieta; nematódeos, pupas de alguns Culicidae e Formicidae estão em segundo lugar, seguido de ostrácodos como as presas menos preferenciais.

Segundo GitTELMAN (1975), ambientes aquáticos maiores são mais estáveis e permitem maior divisão de recursos disponíveis, acompanhado do aumento na habilidade de predadores de diferentes tamanhos utilizarem presas de diferentes tamanhos. No brejo, quando B. platycnemis e Buenoa salutis Kirkaldy, 1904 ocorrem juntas, $B$. platycnemis deve sofrer competição, devido ao pequeno tamanhode $B$. salutis. Esta se favorece na captura de presas de menor tamanho (RIBEIRO et al. 1998). Segundo GITTELMAN (1978), provavelmente alguns Notonectidae que se alimentam de grandes presas são incapazes de otimizar sua dieta à energia requerida para sua sobrevivência. Os custos de perseguição e manuseio da presa aumentam com o decréscimo da relação de tamanho predador-presa, assim como os ganhos energéticos. 


\section{CONCLUSÕES}

Não foram encontradas etapas bem definidas no ciclo populacional de $B$. platycnemis, na área de estudo.

O ciclo de vida dessa espécie parece adaptado ao regime temporário do corpo d'água, embora não tenha sido encontrada nenhuma correlação com a variação do nível d'água.

Foram encontradas posturas em três espécies de macrófitas da área de estudo, mostrando pouca preferência da espécie por uma macrófita específica.

Buenoa platycnemis, em condições não adversas, provavelmente apresenta preferências por organismos com grande mobilidade na coluna d'água. Além disso, a espécie também apresentou uma grande variedade de predadores efetivos na área estudada.

AGRADECIMENTOS. Os autores agradecem aos Profs Luís Fernando M. Dorvillé (Cap, UERJ) e Angela M. Sanseverino (Instituto de Biologia, UFRJ), pelas críticas e revisão do manuscrito. Ao CNPq, à FAPERJ e à FUJB, pela subvenção do estudo.

\section{REFERÊNCIAS BIBLIOGRÁFICAS}

Brower, J.E. \& J.H. Zar. 1977. Field and Laboratory Methods for General Ecology. Dubuque, Wm. C. Brown Pub., 226p.

CARMO, M.A.M. \& L.D. LACERDA. 1984. Limnologia de um brejo de dunas em Maricá, RJ, p. 453-458. In: L.D. Lacerda; D.S.D. ARaújo; R. Cerqueira \& B. TURCQ (Eds). Restingas: origens, estruturas, processos. Niterói, CEUFF, Universidade Federal Fluminense, 582p.

DA SilvA, E.R. DA. 1998. Estratégias de adaptação das espécies de Ephemeroptera às condições ambientais da Restinga de Maricá, Estado do Rio de Janeiro, p. 29-40. In: J.L. NEssimian \& A.L. Carvalho (Eds). Ecologia de insetos aquáticos. Series Oecologia Brasiliensis, V. Rio de Janeiro, PPGE-UFRJ, XVII+309p.

Ellis, R.A. \& J.H. BORDEN. 1970. Predation by Notonecta undulata (Heteroptera: Notonectidae) on larvae of the yellow-fever mosquito. Ann. ent. Soc. Amer. 63 (4): 963-973.

ELLIOTT, J.M. 1977. Some methods for statistical analysis of samples of benthic invertebrates. Ambleside, Freshwater Biological Association, Scientific Publication nbr 25, 160p.

Franco, A.C.; D.M. Valeriano; F.M. Santos; R.P.B. Henriques; J.D. Hay \& R.A. Medeiros. 1984. Os microclimas das zonas de vegetação da praia da restinga de Barra de Maricá, Rio de Janeiro, p. 327-342. In: L.D. LACERDA; D.S.D. ARAúJo; R. CERQUEIRA \& B. TURCQ (Eds). Restingas: origens, estruturas, processos. Niterói, CEUFF, Universidade Federal Fluminense, 582p.

GitTelman, S.H. 1975. The ecology of some Costa Rican backswimmers (Hemiptera: Notonectidae). Ann. ent. Soc. Amer. 68 (3): 511-518.

1978. Optimum diet and body size in backswimmers (Heteroptera: Notonectidae, Pleidae). Ann. ent. Soc. Amer. 71: 737-747.

NESSIMIAN, J.L. 1995a. Abundância e biomassa de macroinvertebrados bentônicos em um brejo de dunas no litoral do Estado do Rio de Janeiro. Rev. Brasil. Biol. 55 (4): 661-683.

- 1995b. Composição da fauna de invertebrados bentônicos em um brejo entre dunas no litoral do Estado do Rio de Janeiro, Brasil. Acta Limnol. Brasiliensis 7: 41-59.

Oliveira, L.; R. Nascimento; L. Krau \& A. Miranda. 1955. Observações biogeográficas e hidrobiológicas sobre a Lagoa de Maricá. Mem. Inst. Oswaldo Cruz 53 (2-4): 171-262.

RiBeIRo, J.R. I.; J.L. Nessimian \& E.C. MENDONÇA. 1998. Aspectos da distribuição dos Nepomorpha (Hemiptera: Heteroptera) em corpos d'água na Restinga de Maricá, Estado do Rio de Janeiro, p.

Revta bras. Zool. 17 (1): 229 - 239, 2000 
113-128. In: J.L. Nessimian \& A.L. Carvalho (Eds). Ecologia de insetos aquáticos. Series Oecologia Brasiliensis, V. Rio de Janeiro, PPGE -UFRJ, XVII+309p.

RICE, L.A. 1954. Observations on the biology of ten notonectoid species found in the Douglas Lake, Michigan Region. Amer. Midl. Nat. 51 (1): 105-132.

SCHNACK, J.A.; E.A. Domizi; A.L. Estévez \& G.R. SPINELLI. 1979. Fecundidad y estructura de edades en una poblacion de Belostoma oxyurum (Dufour) (Hemiptera, Belostomatidae). Revta Soc. ent. argent. 38 (1-4): 11-18.

SCHNACK, J.A.; G.R. SPINELli; A.L. Estévez \& E.A. Domizı. 1987. Ciclo anual y estructura de edades de Belostoma micantulum (Stål) y B. elegans (Mayr), con referencia a dos poblaciones de la provincia de Buenos Aires (Hemiptera: Belostomatidae). Revta Mus. La Plata (n.s.) 14 (156): 83-91.

SOKAL, R.R. \& F.J. RoHLF. 1969. Introduction to Biostatistics. San Francisco, W.H. Freeman \& Co., IX+386p.

TruXaL, F.S. 1953. A revision of the genus Buenoa (Hemiptera -Notonectidae). Kans. Univ. Sci. Bull. 35 (11): 1351-1517.

WigGINS, G.B.; R.J. MACKAY \& I.M. SMITH. 1980. Evolutionary and ecological strategies of animals in temporary pools. Arch. Hydrobiol. Suppl. 58 (1-2): 97-206.

ZALOM, F.G. 1978. A comparison of predation rates and prey handling times of adult Notonecta and Buenoa (Hemiptera: Notonectidae). Ann. ent. Soc. Amer. 71 (1): 143-148.

Recebido em 22.I.1999; aceito em 22.II.2000. 